\title{
Spontaneous Vertebral Artery Dissection and Thrombosis Presenting as Lateral Medullary Syndrome
}

\author{
Neeraj Kumar ${ }^{1}$ Ravindra Kumar Garg ${ }^{1} \quad$ Hardeep Singh Malhotra ${ }^{1} \quad$ Vivek Lal $^{2} \quad$ Ravi Uniyal $^{1}$ \\ Shweta Pandey ${ }^{1}$ Imran Rizvi ${ }^{1}$
}

1Department of Neurology, King George's Medical University, Lucknow, Uttar Pradesh, India

2Department of Neurology, Postgraduate Institute of Medical Education and Research, Chandigarh, Punjab, India

| Neurosci Rural Pract 2019;10:502-503

A 28-year-old male patient had acute-onset headache, vomiting, vertiginous sensation, and diplopia of 3 days' duration. Patient, in the past, had recurrent episodes of migraine. General examination was normal. Cranial nerve examination showed left torsional nystagmus, left Horner's syndrome, decreased pain, and temperature sensation over the left side of the face and decreased pain and temperature sensation over the right side of the body. Power in limbs was normal. Magnetic resonance imaging and computed tomography angiography showed partially thrombosed left
Address for correspondence Ravindra Kumar Garg, MD, DM, Department of Neurology, King George's Medical University, Lucknow 226003, Uttar Pradesh, India (e-mail: garg50@yahoo.com).

vertebral artery aneurysm proximal to the basilar confluence with mass effect and acute infarct on lateral medulla. The patient had left lateral medullary syndrome $(\boldsymbol{-}$ Fig. $\mathbf{1})$. Patient was treated conservatively. The presumed pathogenetic mechanisms for lateral medullary syndrome are large vessel infarction (50\%), arterial dissection (15\%), small vessel infarction (13\%), and cardiac embolism (5\%). In a young patient with migrane, presenting with lateral medullary syndrome, vertebral artery dissection and aneurysm should be suspected. ${ }^{1,2}$

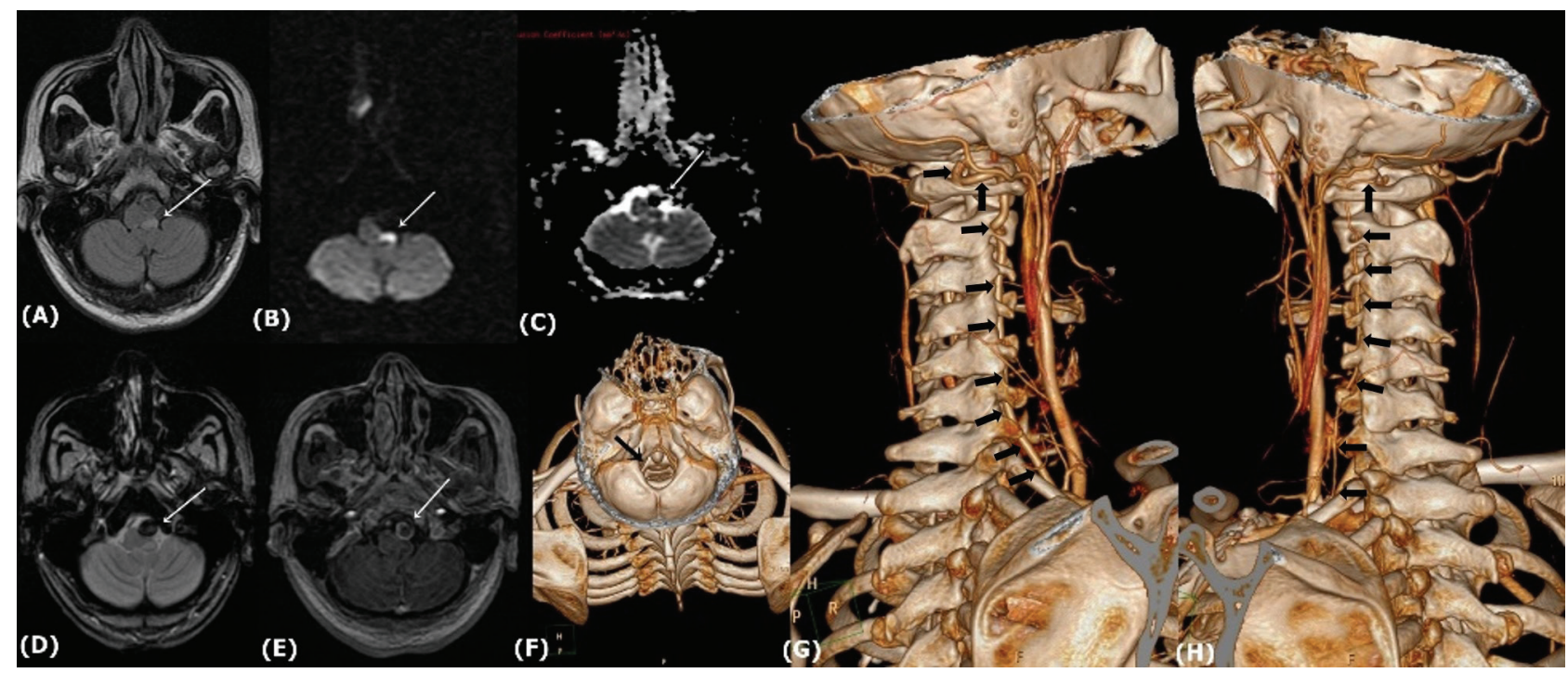

Fig. 1 Magnetic resonance imaging shows left medullary involvement (A). T2 fluid attenuated inversion recovery hyperintensity. (B) Diffusion-weighted imaging restriction. (C) Corresponding apparent diffusion coefficient mapping. (D) Gradient recalled echo blooming in left vertebral artery with contrast enhancement on spoiled gradient contrast. (E) Computed tomography angiography shows absent left vertebral artery. (F) Normal right vertebral artery. (H) Hypoplastic left vertebral artery.

DOI https://doi.org/ $10.1055 / \mathrm{s}-0039-1697243$ ISSN 0976-3147.
License terms

(ㄷ) (1) $\ominus \circledast$ 


\section{Funding}

None.

\section{Conflict of Interest}

None declared.

\section{References}

1 Yen JC, Chan L, Lai YJ. Vertebral artery dissection presented as lateral medullary syndrome in a patient with migraine: a case report. Acta Neurol Taiwan 2010;19(4):275-280
2 Kim JS. Pure lateral medullary infarction: clinical-radiological correlation of 130 acute, consecutive patients. Brain 2003;126(Pt 8):1864-1872 